Source: Khoshnamvand, M., Hao, Z., Fadare, O. O., Hanachi P., Chen, Y., \& Liu, J. (2020). Toxicity of biosynthesized silver nanoparticles to aquatic organisms of different trophic levels. Chemosphere, 258. doi:10.1016/j.chemosphere.2020.127346. https://doi.org/10.1016/j.chemosphere.2020.127346

\title{
Toxicity of biosynthesized silver nanoparticles to aquatic organisms of different trophic levels
}

Mehdi Khoshnamvand ${ }^{1}$, Zhineng $\mathrm{Hao}^{2}$, Oluniyi O Fadare ${ }^{3}$ Parichehr Hanachi $^{4}$, Yongsheng Chen $^{5}$, Jingfu Liu ${ }^{6}$

${ }^{1}$ State Key Laboratory of Environmental Chemistry and Ecotoxicology, Research Center for Eco-Environmental Sciences, Chinese Academy of Sciences, Beijing, 100085, China; University of Chinese Academy of Sciences, Beijing, 100049, China. Electronic address: mehdi.khoshnam@yahoo.com.

${ }^{2}$ State Key Laboratory of Environmental Chemistry and Ecotoxicology, Research Center for Eco-Environmental Sciences, Chinese Academy of Sciences, Beijing, 100085, China. Electronic address: znhao@rcees.ac.cn.

${ }^{3}$ State Key Laboratory of Environmental Chemistry and Ecotoxicology, Research Center for Eco-Environmental Sciences, Chinese Academy of Sciences, Beijing, 100085, China; University of Chinese Academy of Sciences, Beijing, 100049, China. Electronic address: fadare@ rcees.ac.cn.

${ }^{4}$ Department of Modern Sciences and Technologies, Faculty of Medicine, Mashhad University of Medical Sciences, Mashhad, Iran

${ }^{5}$ School of Civil and Environmental Engineering, Georgia Institute of Technology, Atlanta, GA, 30332, USA. Electronic address: yongsheng.chen@ce.gatech.edu.

${ }^{6}$ State Key Laboratory of Environmental Chemistry and Ecotoxicology, Research Center for Eco-Environmental Sciences, Chinese Academy of Sciences, Beijing, 100085, China; Institute of Environment and Health, Jianghan University, Hubei Province, Wuhan, 430056, China. Electronic address: jfliu@ rcees.ac.cn.

\begin{abstract}
Although biosynthesized nanoparticles are regarded as green products, research on their toxicity to aquatic food chains is scarce. Herein, biosynthesized silver nanoparticles (Alcea rosea-silver nanoparticles, AR-AgNPs) were produced by the reaction of Ag ions with leaf extract of herbal plant Alcea rosea. Then, the toxic effects of AR-AgNPs and their precursors such as Ag+ ions and coating agent (A. rosea leaf extract) on organisms of different trophic levels of a freshwater food chain were investigated. To the three studied aquatic organisms including phytoplankton (Chlorella vulgaris), zooplankton (Daphnia magna) and fish (Danio rerio), the coating agents of AR-AgNPs showed no toxic effects, and Ag+ ions were more toxic in comparison to ARAgNPs. Further investigations revealed that the release of Ag+ ions from AR-AgNPs to the test media were not considerable due to the high stability of AR-AgNPs, thus the toxicity stemmed mainly from the particles of AR-AgNPs in all the three trophic levels. Based on values of 72-h EC50 for C. vulgaris, 48-h LC50 for D. magna and 96-h LC50 for D. rerio, the most sensitive organism to AR-AgNPs exposure was D. magna (the second trophic level).
\end{abstract}

Keywords: Toxicity, Silver nanoparticles, Biosynthesized nanoparticles, Aquatic organisms, Trophic level. 